\title{
Inner Processes of Photon Emission and Absorption
}

\author{
Dirk J. Pons ${ }^{1}$ \\ ${ }^{1}$ Department of Mechanical Engineering, University of Canterbury, New Zealand \\ Correspondence: Dirk Pons Department of Mechanical Engineering, University of Canterbury, Private Bag 4800, \\ Christchurch 8020, New Zealand. Tel: 64-3364-2987. Email: dirk.pons@canterbury.ac.nz
}

Received: June 7, 2014 Accepted: June 17, 2015 Online Published: July 14, 2015

doi:10.5539/apr.v7n4p24 URL: http://dx.doi.org/10.5539/apr.v7n4p24

\begin{abstract}
Problem- There are deep unanswered questions about photon emission, specifically how the field structures of the photon emerge from the electron. While this problem cannot be answered from within quantum mechanics, due to its premise that particles are zero dimensional points, other theories of physics have better prospects. Purpose- A conceptual theory is developed for the processes of photon emission and absorption, in a non-local hidden-variable (NLHV) design called the Cordus theory. Approach- Logical inference is used to predict the structures of the discrete fields of the photon and electron under this framework. From this is extracted an explanation of how electron bonding constraints require the electron to emit its excess energy, and how the photon is created and emerges from the electron. Findings- Emission is found to be an escapement mechanism whereby matter particles that are over-prescribed in position can get rid of that energy, and the details of this are explained. The results show excellent qualitative agreement with classical electromagnetic wave theory and quantum mechanics, many conceptual features of which can be recovered. Originality- A novel conceptual theory is developed for the processes of photon emission and absorption. Particularly important here is the proposed causality whereby bonding constraints affect geometric span of the particule, which affects frequency. Hence this constrains the energy that the electron can contain, and explains why the emitted photon has a specific quantum of energy. A second contribution is the proposed differentiation between the discrete fields of the photon and electron. This yields an explanation for the exponential nature of the evanescent field, and recovery of the inverse radius squared relationship for the electro-magneto-gravitational fields. The theory also offers a physical interpretation of the fine structure constant $\alpha$ as a measure of the transmission efficacy of the fabric, i.e. $\alpha$ determines the relationship between the electric constant of the vacuum fabric, and the speed of propagation $\mathrm{c}$ through the fabric. There is further novelty in achieving this from the non-local hidden-variable sector of physics. Implications- Quantum mechanics originated with the observation that the movement of electrons between orbitals resulted in emission of photons with discrete quanta of energy. However it does not explain how the transitions occur. The original contribution here is showing that a solution does exist in NLHV physics. Assuming this is valid then the implications are that particles have internal structure and that quantum mechanics is merely a quantitative statistical summary of a deeper physics.
\end{abstract}

Keywords: light, electron, absorption, emission, evanescent field, physical realism

\section{Introduction}

Our world is full of light and its interactions with matter. Yet describing how photons are emitted and absorbed is surprisingly difficult. The mathematical formulations of quantum mechanics (QM) and electromagnetic wave theory are good at quantifying what happens, but not at describing how the photon emerges from the electron at emission, or is absorbed back into it. Even asking how an electron physically emits a single photon is a meaningless question from the zero-dimensional (0D) point perspective of QM, and wave theory does not extend to single particles. So the question remains: How is a photon emitted from an electron? And the complementary question: How is light absorbed by matter?

The purpose of this work is to explore these fundamental questions about the physical process of photon emission and absorption. We show that it is possible to develop a new way of understanding this problem, using the Cordus theory as the starting point, and some specific structures for the electron and photon. We present a novel conceptual theory of the processes for emission and absorption. The particular area of examination is the interaction of a photon and an electron. We propose processes for how the photon emerges from, or is absorbed, into the electron. 


\section{Background}

The question here concerns the process by which photon emission occurs. The existing approaches to this fall into several categories. The most basic underlying principles are in terms of quantum changes in energy levels. From this emerge various rate constants (or Einstein coefficients) for the likelihood of transition in the situation under examination. However a fuller explanation required a second quantification for quantum mechanics, that of the electromagnetic field, hence quantum electrodynamics (QED) (Dirac, 1930). The explanatory principle underlying this approach is that the vacuum electromagnetic field introduces a (small) perturbation to the quantum state, which initiates the transition to emission. Perturbation theory with renormalisation may then be used to enumerate the possible routes to the outcomes. In this way the emission rates can be given an explanation (Cohen-Tannoudji, Dupont-Roc, \& Grynberg, 1998; Loudon, 2001). The existing literature is well-endowed with specific case studies of describing what emission effects are observed for various combinations of situational factors and base materials. The how side of the process has received some attention, including near-field electrodynamics (Keller, 2006), field of the emerging photon (Keller, 2000), interaction with the vacuum fields (Alexanian \& Bose, 2006), and coherent states (Zavatta, Viciani, \& Bellini, 2005). For most purposes, this is enough explanation for empirical work. However the ontological questions remain: How does the field structure of the photon emerge from that of the electron? There is a need for theories that better explain the photon emission and absorption processes.

\section{Purpose and Approach}

The purpose of this work was to attempt an explanation of photon emission with a design from the non-local hidden-variable (NLHV) sector. This sector has generally been disfavoured by theorists, and would generally be considered a non-useful starting position. This is because the Bell type inequalities (Bell, 1964; Groblacher et al., 2007; Leggett, 2003) invalidate local hidden-variable designs. Non-local designs are not precluded, but none have previously been found other than the de-Broglie-Bohn theory (de Broglie, 1925). However that has not progressed beyond a narrow area of application. Nonetheless recent conceptual work in the NLHV sector has shown that there is a potentially viable design, and this is the Cordus theory (named for the shape it predicts for particles). A whole new physics emerges from this idea, and includes solutions for wave-particle duality (D. J. Pons, A. D. Pons, A. M. Pons, \& A. J. Pons, 2012), unification of the electro-magneto-gravitational and strong forces (D. J. Pons, A. D. Pons, \& A. J. Pons, 2013b), explanations for antimatter and the process of annihilation (D. J. Pons, A. D. Pons, \& A. J. Pons, 2014c) (D. J. Pons, A. D. Pons, \& A. J. Pons, 2014a), a solution that explains the table of nuclides (H to Ne) (Pons, Pons, \& Pons, 2013a), and a theory for time (D. J. Pons, A. D. Pons, \& A. J. Pons, 2013c). The present paper shows that the theory may be extended to also explain photon emission.

This conceptual development was achieved using a systems-engineering design methodology. The systems engineering part of the methodology ensures that the development of the sub-theory for emission is logically consistent with the overall theoretical framework. The design part of the methodology is used to infer the requisite internal structures of a system, from its known external behaviour or required functionality. The design process is an iterative development that creates new concepts, evaluates them for fitness, i.e. the extent to which the concept successfully explains known features of emission, and changes and refines the concepts accordingly.

The approach was to start with the NLHV design provided by the Cordus theory (D. J. Pons, A. D. Pons, A. M. Pons, \& A. J. Pons, 2012), which is summarised below. This also includes a theory for the emission of discrete forces, i.e. the fields are proposed to be discretised (D. J. Pons, A. D. Pons, \& A. J. Pons, 2013b). Other work has shown that under this set of assumptions, particles would be defined by the attributes of the discrete forces they emit, and that changing those discrete forces transmutes the particle, hence explanations for annihilation (D. J. Pons, A. D. Pons, \& A. J. Pons, 2014a), asymmetrical baryogenesis (D. J. Pons, A. D. Pons, \& A. J. Pons, 2014b), and nuclear decay processes (Pons, Pons, \& Pons, 2015a, 2015b, 2015c). The emission problem may then be reformulated as a need to describe how the discrete force structures of the photon emerge from those of the electron. The structures predicted by the Cordus theory for the photon and electron were input to the design method, to infer the requisite emission processes. Thereafter the photon-absorption process were checked, and these were found to be symmetrical to the emission process. Many conceptual iterations were performed but only the final results are shown below. The ontological questions, e.g. why the electron needs to emit a photon in the first place, are then addressed.

\section{Results}

We start with a brief summary of the Cordus theory, as this is not widely known. We then differentiate the discrete field structures of the photon and electron, and then propose a mechanism for photon emission in terms of these discrete fields. 


\subsection{Cordus Theory}

The core conjecture of the Cordus theory is that all particles have inner and outer structures comprising two reactive ends some distance apart (span), connected by a fibril (hence cordus), and emitting discrete forces (D. J. Pons, A. D. Pons, A. M. Pons, \& A. J. Pons, 2012). This is called a particule to differentiate it from the zero-dimensional (0D) point idea of quantum mechanics (QM). The fibril is a persistent structure that provides instantaneous connectivity and synchronicity between the two reactive ends, but does not interact with matter. The reactive ends are energised sequentially (at the de Broglie frequency), during which they emit discrete forces out into the external environment. The locus of these over time defines a flux line called a hyperfine fibril (hence hyff). The discrete forces are emitted in three spatial directions (hence hyff emission directions, HEDs), and hence space is filled with a fabric of discrete forces (D. J. Pons \& A. D. Pons, 2013). The quantity, direction, and arrangement of these discrete forces determine the type of particule and are responsible for charge, mass, matter-antimatter species differentiation, and spin (D. J. Pons, A. D. Pons, \& A. J. Pons, 2014c). The discrete forces are responsible for the electro-magneto-gravitational and strong interactions, though the theory uses the term synchronous interaction in place of the strong, as this better describes the proposed nature of the interaction (D. J. Pons, A. D. Pons, \& A. J. Pons, 2013b). The next section applies this theory to the photon and electron.

\subsection{Photon Structure}

Within this theory the photon is required to have an oscillating system of discrete fields, see Figure 1. In contrast to massy particules, the photon reactive end pumps out a discrete force, and then promptly withdraws it, never releasing it. The outward motion corresponds to negative charge, and inward to positive (a sign convention). Thus the photon changes sign. This is consistent with the observation that the electric field of the photon reverses sign.

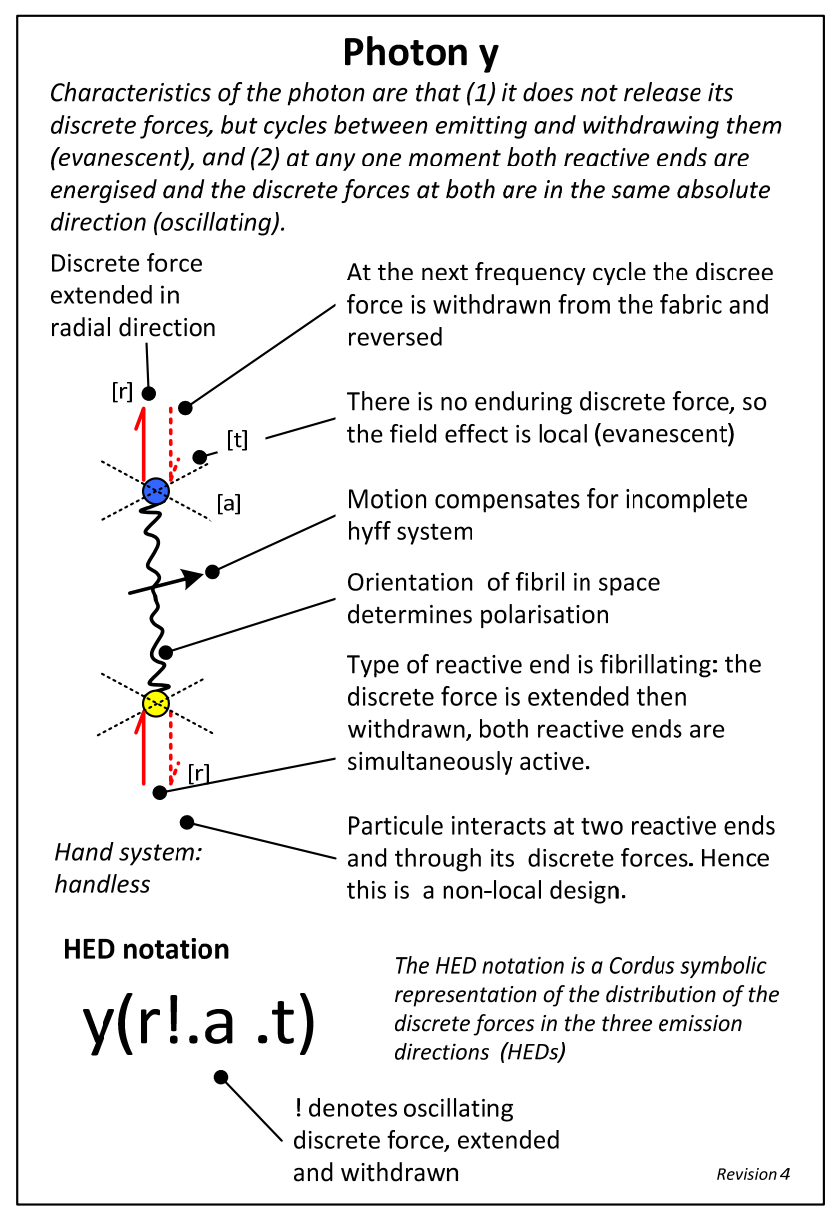

Figure 1. Cordus theory for the internal structure of the photon, and its discrete field arrangements. The photon has a pump that shuttles energy outwards into the fabric. Then at the next frequency cycle it draws the energy out of that field, instantaneously transmits it across the fibril, and expels it at the opposite reactive end. From (Pons et al., 2014a) with permission 


\subsection{Electron Structures}

This theory requires that the electron (along with all matter and antimatter), pushes a discrete force pulse outwards along a persistent hyff, or pulls inwards in the case of positive charge, see Figure 2. The hyff is enduring, and the direction of propagation of the discrete forces is consistently either medially or distally at both reactive ends, in contrast to the photon. The discrete forces are force pulses transmitted along the hyff lines, and remain connected to the reactive end at the base. Each frequency cycle sends out another set of discrete forces. Hence we call this a pulsating pump.

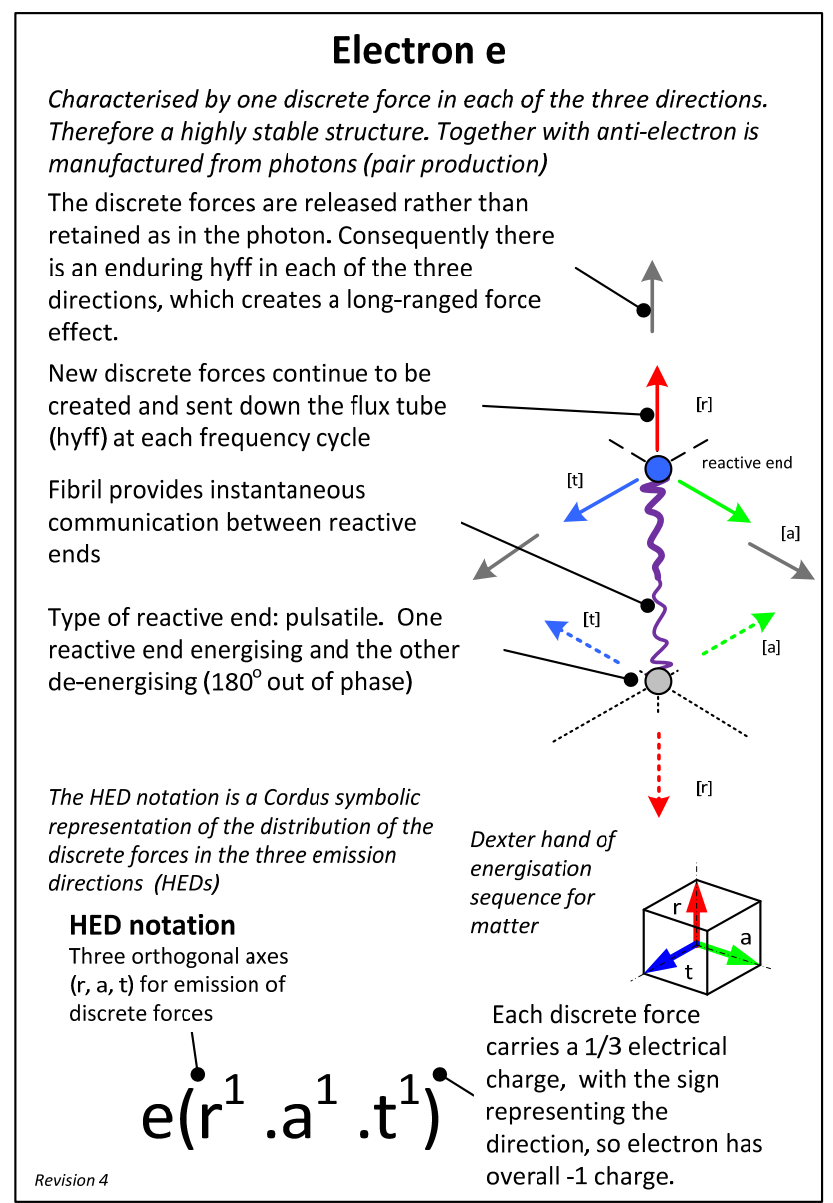

Figure 2. Cordus theory for the internal structure and external field arrangements of the electron. The electron pushes discrete forces (fragments of electric field) consistently outwards in a pulsating manner. All other matter and antimatter behaves like the electron, though the direction of field propagation is reversed for positive charge, and the energisation sequence (hand) is inverted for antimatter. From (Pons et al., 2014a) with permission

\subsection{Evanescent and Electric Fields}

This Cordus field theory is also consistent with the known ranged effects of the two particules, and explains why there is a difference. The photon is known to have only a short range for its electromagnetic (EM) field: the strength drops off exponentially with distance. In optics this is termed the evanescent wave.

\subsubsection{Evanescent Field}

The Cordus explanation is that the photon pushes its discrete force into the local external space where its effect is absorbed into the volume of space. The exponential nature of the distribution arises because the relative absorption of the energy into the space is constant with radius. Another way of explaining this is that the photon pushes its energy into the entire local volumetric supply-chain of space. Elsewhere it has been identified that, under the Cordus theory, space consists of a fabric comprising the discrete forces of all the other massy particules in the accessible universe (Pons \& Pons, 2013). Thus a third explanation is that the photon pushes its energy into a 
transient disturbance of the surrounding fabric discrete forces. This last explanation is consistent with that from wave-theory, which also considers the photon as a self-propagating field-disturbance. These explanations are all complementary.

The photon can be considered to generate a volumetric strain in the fabric, with a constant differential in the radial direction. This strain is created at each of its two reactive ends. The absorption into space is elastic, and at the next frequency cycle the photon receives the energy back from the fabric and transmits it out of the other reactive end. Thus the fabric of space has a temporal impedance.

\subsubsection{Fine Structure Constant $\alpha$}

The impedance of free space is $Z_{0}=1 /\left(\varepsilon_{0} c\right)=2 \alpha h / \mathrm{e}^{2}$, with electric constant $\varepsilon_{0}$ (also called vacuum permittivity), the speed of light in the vacuum $\mathrm{c}$, and the fine structure constant $\alpha=\mathrm{e}^{2} /\left(2 \varepsilon_{0} \mathrm{hc}\right)$, with elementary charge e [coulombs], Planck constant $\mathrm{h}$, and $\mathrm{c}$ as before. All these are generally considered physical constants. In contrast the Cordus theory proposes that both the vacuum permittivity and the speed of light are dependent variables, and situationally specific. It is proposed that $\varepsilon_{0}$ represents the density of the discrete forces in the fabric, and thus depends on the spatial distribution of mass within the universe. Thus the electric constant is recast as an emergent property of the fabric.

From this perspective $\alpha$ is a measure of the transmission efficacy of the fabric, i.e. it determines the relationship between the electric constant of the vacuum fabric, and the speed of propagation $\mathrm{c}$ through the fabric. This is consistent with the observation that $\alpha$ appears wherever electrical forces and propagation of fields occur, and this includes cases such as electron bonding. The reason the speed of light is limited to a certain finite value is explained as a consequence of the fabric density creating a temporal impedance. Thus denser fabric results in a slower speed of light, and this is consistent with time dilation (Pons et al., 2013c), and optical refraction generally. In the Cordus theory the speed of light in turn is determined by the density of the fabric discrete forces and is therefore locally consistent and relativistic, but ultimately dependent on the past history of matter density in the locally available universe. Thus the vacuum (fabric) has a finite speed of light, despite an instantaneous communication across the fibril. This Cordus theory is consistent with the known impedance of free space though comes at it from a novel direction.

\subsubsection{Electric Field}

The electron has a much greater range for its electromagnetic fields - potentially infinite - and though they do drop off with radius squared it is not as quickly as the photon's. Indeed all of the electro-magnetic-gravitational (EMG) fields reduce this way. The Cordus explanation is that all three EMG forces are carried by discrete forces, as tension, bending and torsion (handedness) respectively of the flux tube containing the discrete forces (D. J. Pons, A. D. Pons, \& A. J. Pons, 2013b). Thus one underlying element, the discrete force, is proposed as the mechanism for all these interactions.

The conventional explanation for the radius-squared reduction of the EMG forces is the inverse-square law. This states that the fields are conserved and therefore spread over the surface area of an expanding sphere as they propagate outwards. The Cordus theory agrees with this explanation, but can add another level of detail: the discrete forces themselves are discrete as the name suggests, and produced at the frequency of the massy particule. So for a given body and frequency (hence mass or energy) the rate of discrete force production is constant, and hence the total number available is conserved as they propagate outwards over the area of the sphere. In addition, the Cordus theory predicts that the discrete forces, and hence also the fields, are not consumed by interaction with remote matter particules, but instead continue to propagate outwards indefinitely, i.e. they cannot be shielded.

\subsubsection{Comparison}

According to this theory, the photon's discrete forces are pushed into (or pulled from) a recruited volume of space and impeded in the process, hence the exponential reduction with range. In contrast the electron's discrete forces are propagated outwards as a series (flux line) that is not weakened with distance, and therefore their density is diluted across the surface of a growing sphere. Hence a reduction with radius-squared for all the EMG forces. The difference really comes down to the photon needing to borrow discrete forces from the fabric, whereas the electron contributes discrete forces to make the fabric.

If we were disembodied spirits contemplating a physics that was being designed but yet to be built, we might naturally expect all fields to recruit the full volume of space they traverse and thus reduce exponentially with radius. That the EMG fields do not, despite propagating into the same medium as those of the photon, shows that there is something profoundly different and unusual about their mechanisms. We are so used to seeing the $1 / \mathrm{r}^{2}$ relationship in the EMG fields that we take it as the norm and perceive the exponential relationship of the photon 
evanescent field as the anomaly, when perhaps it is more helpful to take the inverse position. This characteristic of the EMG fields strongly suggests that the field elements are discrete (i.e. discrete forces for Cordus, or bosons for $\mathrm{QM}$ ), are diluted over an expanding front, only affect other objects as that front passes through, are not consumed in the usage, and cannot be shielded. The cumulative effect of many small such interactions is the electro-magnetic-gravitational field, and hence electromagnetic wave theory is interpreted as the coarse overall representation of a deeper discrete effect, just as the QM wave function becomes an average representation of a deeper determinism in particule location.

We now have a theory for the structure of the photon and electron, comprising the internal structure and the external field features. Next we use this to develop an explanation of the mechanics of photon emission.

\subsection{Photon Emission Process}

How does the electron absorb and emit a photon? The previous works of the Cordus conjecture have established some general principles for photon absorption, Compton scattering, mid-energy interactions, photoelectric effect, heat, and photon emission. However that was in general terms, and did not go into the possible deeper mechanics at the discrete force level, which is the present purpose. Here we explore the possible finer behaviours of the Cordus structures of the electron and photon as they interact.

\subsubsection{Theory of Photon Release}

We start with an electron that has excess energy. We anticipate several pathways for this state to arise:

- The electron immediately previously absorbed a photon.

- The electron received energy from other particules with which it was bonded. The Cordus theory proposes that assemblies of matter that are bonded by the strong force, which is to say coherent, will share energy throughout the assembly.

- The electron changed its bonding configuration to one that needs less energy, so a surplus exists. The Cordus theory is that bonding involves particules with compatible geometry and frequency. So a change to a bond causes the span to change. In turn this causes the frequency to change, and thus changing the energy that the electron is allowed to contain. The inverse proportionality between frequency (and energy) to span is a Cordus concept, not accessible to theories like QM that are based on 0D points.

We also anticipate that the electron is constrained by bonding, at least to some extent. Thus a free electron is able to accommodate greater or lesser amounts of energy, simply by adjusting its frequency and span. However an electron that is bonded has less freedom, or even none, to make these adjustments and must therefore either dispose of the energy or break the assembly. Those assembly forces are variants of the synchronous interaction (strong force). This is consistent with the empirical observation that sufficiently energetic electrons will break out of their assemblies, hence ionising radiation, whereas less energetic cases result in photon emission or temperature rise. The thermal case is explained in the Cordus theory as a phonon effect, and not discussed further here as our interest lies with the photon emission category.

At a systems level this Cordus theory is represented using the systems engineering modelling notation of integration definition zero (IDEF0) (FIPS, 1993). The IDEF0 model represents the proposed relationships of causality, and thus qualitatively serves the same purpose as mathematical formalism does in conventional physics. With IDEF0 the object types are inputs, controls, outputs, and mechanisms (ICOM) and are distinguished by placement relative to the box, with inputs always entering on the left, controls above, outputs on the right, and mechanisms below. The overall theory for emission is shown in Figure 3. There are four stages in this process, elaborated below.

\subsubsection{Energy Determines Target Re-Energisation Locations for Electron (1)}

The process of emission is proposed to operate as follows. The initial state of an energised but constrained electron is shown in Figure 4. The electron may be fixed at one or both reactive ends (REs) by bonding arrangements, not shown, which occur through its discrete forces. These constraints mean that the discrete forces are expected to energise in a particular place, time, and phase. This because the nature of bonding requires synchronicity of emissions (Pons et al., 2013a). The temporary higher energy state of the electron nominally requires that it move to a higher frequency and shorter span. The changed frequency cannot be accommodated by the bond commitments. The shorter span results in target re-energisation locations as shown, though these too are not achievable. 


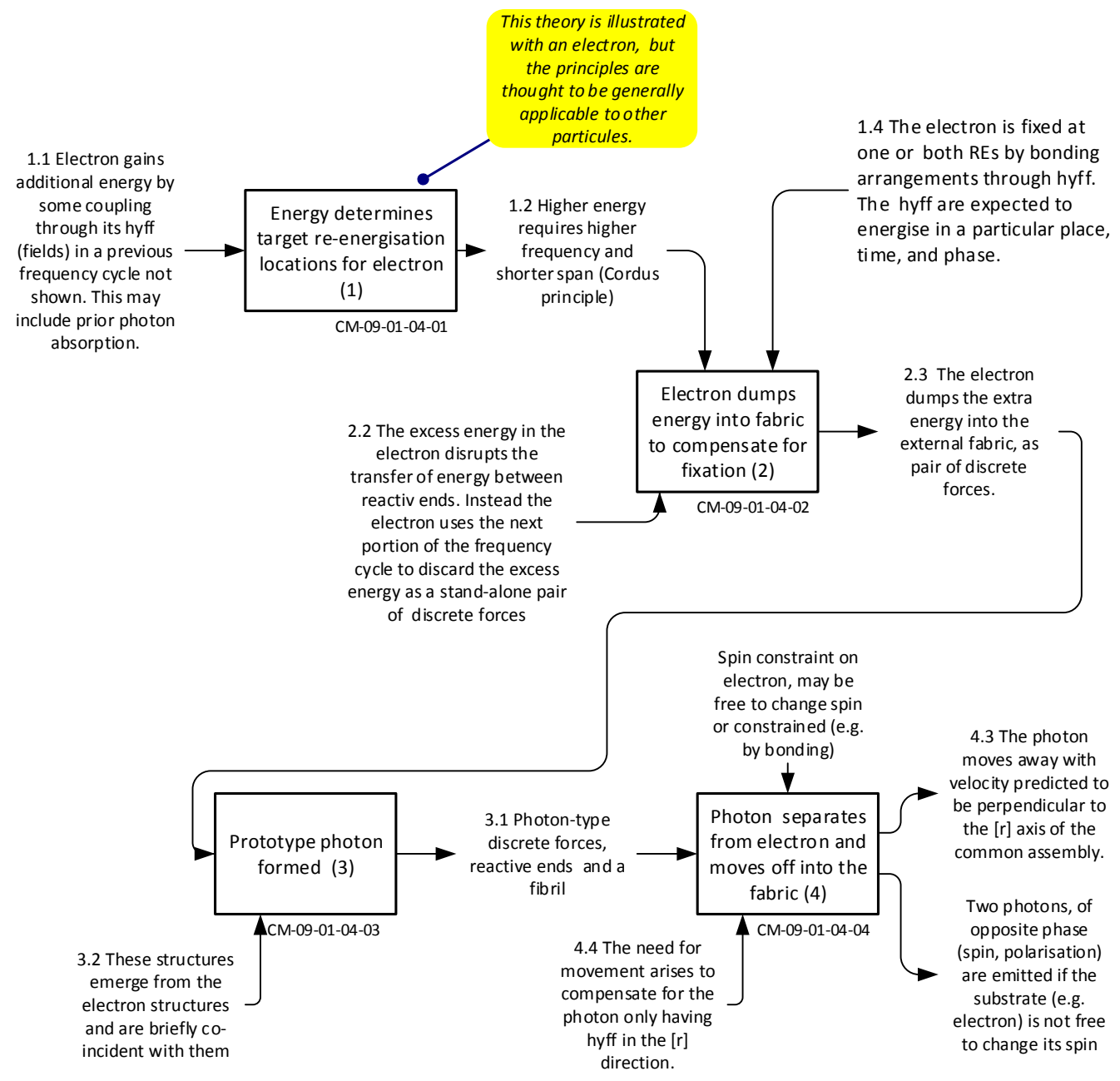

Figure 3. Systems representation of the overall theory for photon emission

CM-09-01-04-01

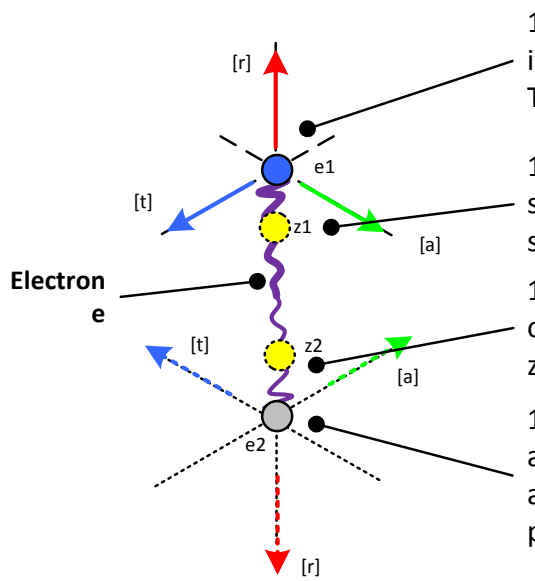

Figure 4. Energetic electron shown. $\mathrm{z} 2$ are symmetrical. phase.

\section{Energy determines target re-energisation locations for electron}

1.1 Electron gains additional energy by a coupling through its discrete fields in a previous frequency cycle not shown. This may include prior photon absorption.

1.2 Higher energy requires higher frequency and shorter span (Cordus principle). Target re-energisation location z1

1.3 The fibril instantly communicates the distress to the other reactive end. Consequently target RE locations $z 1$ and

1.4 But the electron is fixed at one or both REs by bonding arrangements through discrete forces. The discrete forces are expected to energise in a particular place, time, and 


\subsubsection{Electron Dumps Energy into Fabric to Compensate for Fixation (2)}

To compensate for these constraints, the electron dumps the extra energy into the external fabric, where it is temporarily taken up as a volumetric strain, and appears as a photon discrete force, see Figure 5. Off-loading this energy allows the reactive end to stay at its current location, and preserve its frequency. The second reactive end does likewise, because the instantaneous communication of the fibril keeps the two ends synchronised. The second $\mathrm{RE}$ therefore also creates a volumetric strain in the fabric, but of the opposite charge. This nonetheless also requires energy. So half the excess energy goes into each of the two newly created fields. These fields are of the exponential type as they involve progressive recruitment of a volume of fabric (Note 1).

\section{CM-09-01-04-02}

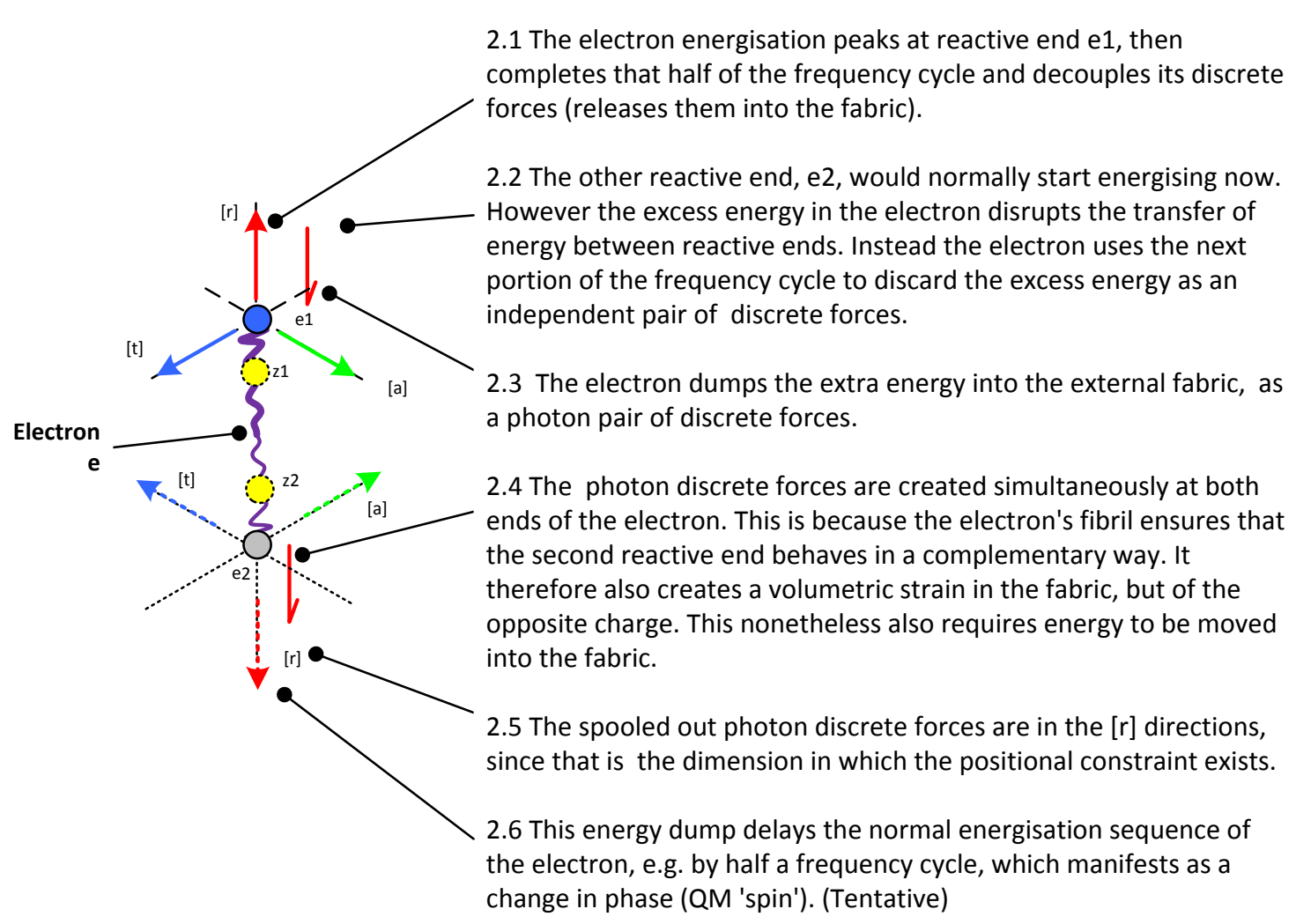

\section{Electron dumps energy into fabric to compensate for fixation}

2.1 The electron energisation peaks at reactive end e1, then completes that half of the frequency cycle and decouples its discrete

2.2 The other reactive end, e2, would normally start energising now. However the excess energy in the electron disrupts the transfer of energy between reactive ends. Instead the electron uses the next portion of the frequency cycle to discard the excess energy as an

Figure 5. Electron dumps energy

The Cordus theory for annihilation of positronium made an assumption that photon emission changes the phase of the electron (Pons et al., 2014a). This effect also applies when the electron is being accelerated (includes deceleration) or forced to change its orientation (spin). We suspect that this may be universal to photon emission, i.e. that the electron changes phase in all situations where it emits a photon, though we cannot be certain (Note 2). The proposed mechanism for this is provided at the energy dump stage, see Figure 5, note 2.6. We predict that two photons, of opposite phase (spin, polarisation) are emitted if the electron is not free to change its spin, otherwise one.

\subsubsection{Prototype Photon Formed (3)}

A prototype photon then forms, as new reactive ends and fibril emerge from the electron structures to support the new discrete forces. The photon only needs a single fibril, which also makes up the discrete forces at each end, so the reactive ends are not as distinct as for the electron. The new fibril is of the oscillating type. These photon structures are briefly co-incident with those of the electron, see Figure 6.

\subsubsection{Photon Separates from Electron and Moves Off into the Fabric (4)}

The photon moves away with velocity perpendicular to the $[\mathrm{r}]$ axis of the common assembly, see Figure 7 . The need for movement arises as a stability requirement to compensate for the photon only having discrete forces in the $[\mathrm{r}]$ 
direction. Thus the orientation of the original electron particule determines the direction of release of the photon. This theory tentatively also predicts that the direction of motion of the photon in the $[\mathrm{a}, \mathrm{t}]$ plane would be determined by the energisation sequence of the electron discrete forces, i.e. the orientation. However, other than saying the photon would be emitted in the [a.t] plane, we cannot yet be more specific. We leave this as an open question.

\section{CM-09-01-04-03 3 Prototype photon formed}

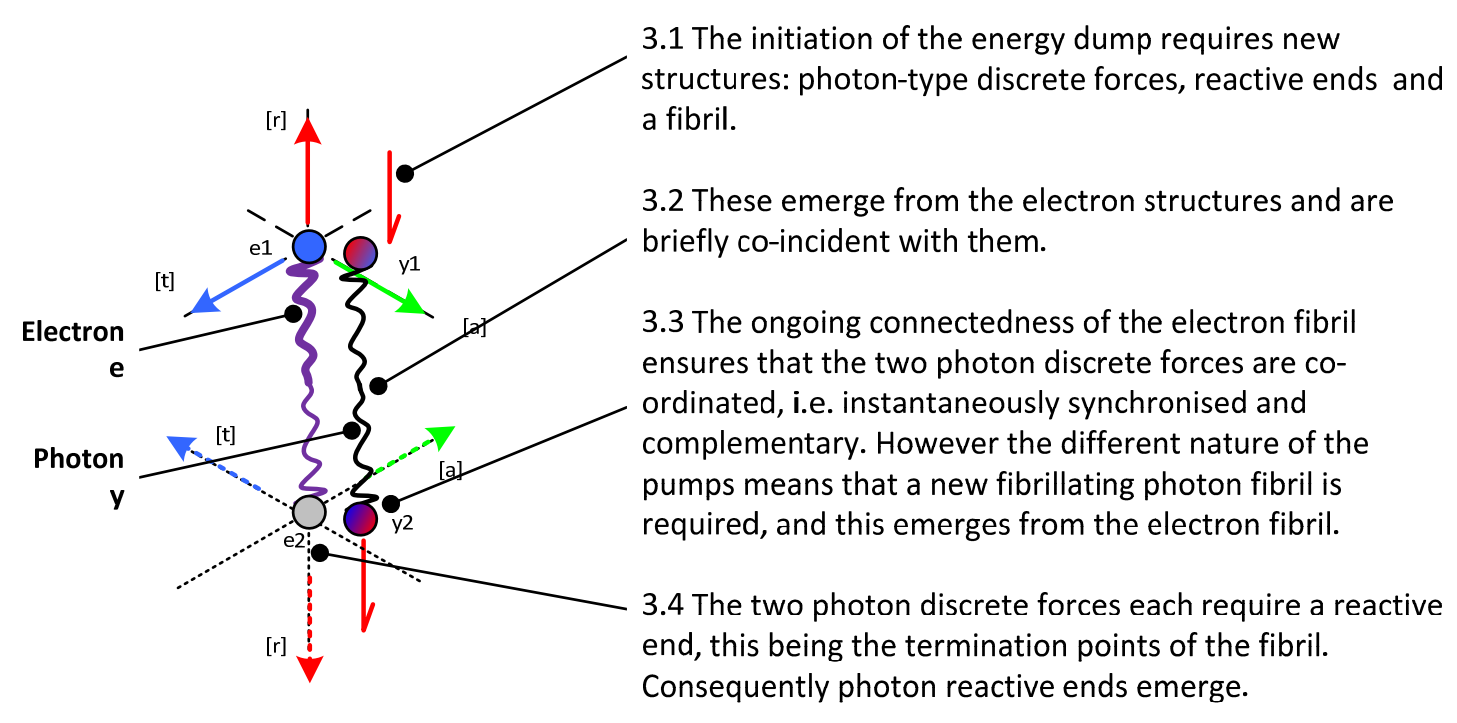

3.5 Electron span may change in the process, e.g. the span of a free energetic electron is expected to lengthen as it releases a photon.

Figure 6. Prototype photon forms

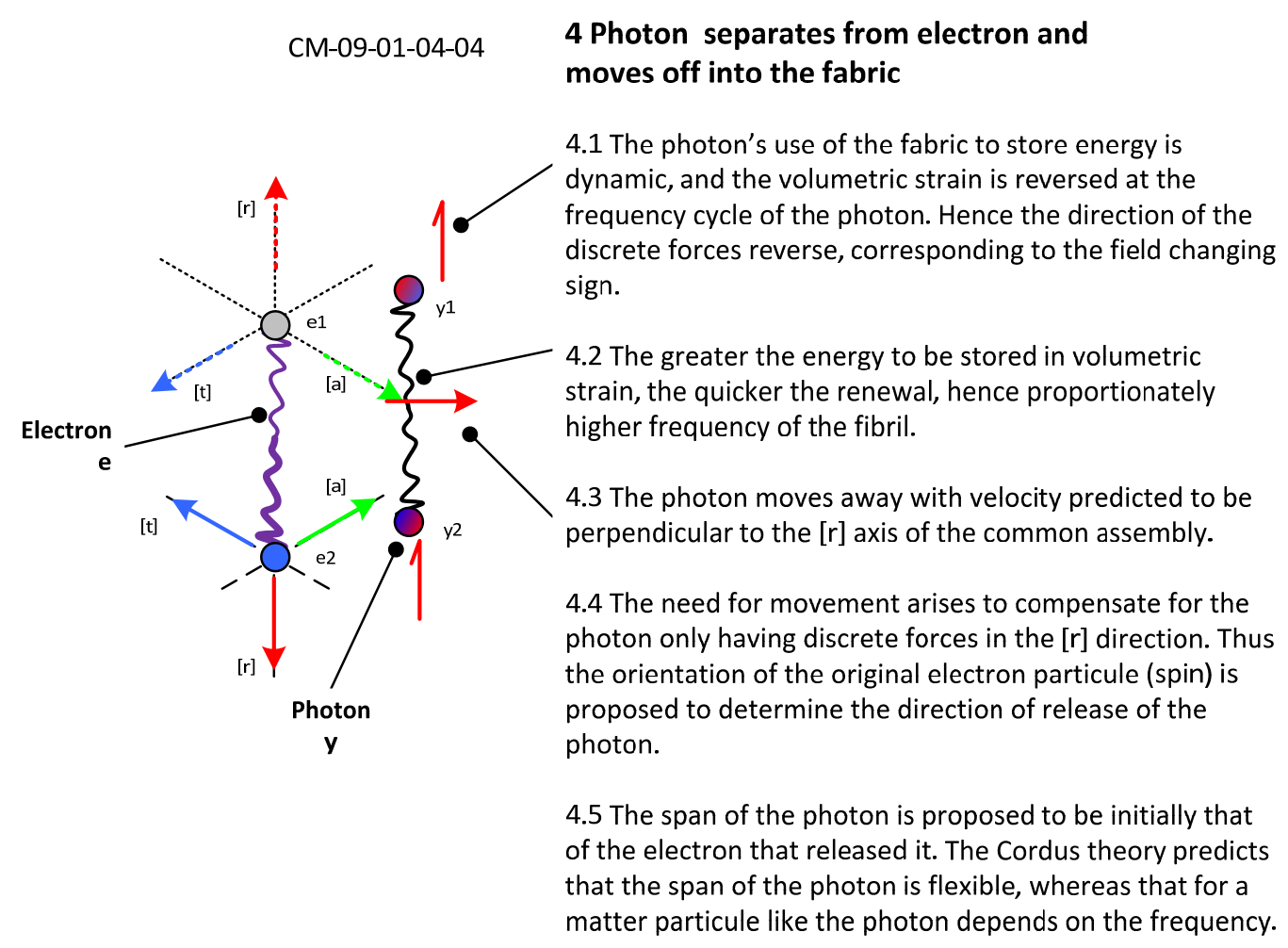

Figure 7. Photon separates from electron and moves off into fabric 
The photon's use of the fabric to store energy is dynamic, and the volumetric strain is reversed at the frequency cycle of the photon. The velocity of the photon is determined by the impedance of getting its discrete force energy into and extracted from the fabric and thus limited to the speed of light in the medium.

This explanation suggests that the span of the photon is likely initially that of the electron that released it. The Cordus theory requires the span of the photon to be flexible, being decoupled from the frequency, whereas the span for any massy particule is inversely linked to frequency. This is also consistent with the Planck relationship for energy, $\mathrm{E}=\mathrm{hf}$ with Planck constant $\mathrm{h}$, and frequency $\mathrm{f}$. The Cordus explanation is the greater the energy to be stored in volumetric strain, the quicker the renewal needs to be. Hence higher energy photons have proportionately higher frequency of the fibril.

\subsection{Photon Absorption Process}

The above describes the proposed process of photon emission. It is now possible to provide a process for the absorption of the photon into the electron. We do this by running the above process in reverse, on the assumption that the processes are symmetrical.

The photon discrete force, which is one axis only [r], comes within range of the discrete forces of the electron, and starts to interact with them. The interaction is initially only within the wider fabric: the electron is contributing enduring discrete forces to the fabric, and the photon is creating transient discrete force disturbances within that same fabric. Therefore the hyff systems of the two particules need to be interacting, but the particules themselves as defined by their reactive ends, do not need to be coincident. This is part of the non-local nature of this theory. It may not matter precisely which HED of the electron engages with the photon hyff, because they can likely redistribute the energy into the rest of the particule. The next stage is a decision point as to which process the particules follow. The options are absorption, partial energy transfer, disturbance, deflection, reflection, or no interaction. We propose this is deterministic, and depends on the relative orientation of the two particules, their proximity, momentum, relative phases, and frequency. Thus there are more variables available than in the conventional $0 \mathrm{D}$ point theory of $\mathrm{QM}$. We anticipate that for absorption the particules need to be at least somewhat aligned so that the four reactive ends can sense each other's hyff. The respective reactive ends also need to be in phase. Continuing the absorption process, the electron pulls the photon into final alignment and synchronicity, via the hyff. We assume that the mechanisms are flexible enough to accommodate some degree of initial mismatch. We also assume that the photon span is capable of changing length to match the electron span. It may take several frequency cycles to achieve sufficient match, hence the possibility that absorption does not occur but some degree of energy transfer does. Once the electron and photon reactive ends are sufficiently coincident, the energy of the photon, which is dynamic, is captured by the electron through the hyff structures. The photon structures (fibril and reactive ends) collapse or are absorbed into the electron. A phase change is presumably involved here, if it applies at emission. The general sequence is proposed to be the reverse of the figures previously given for emission.

\section{Discussion}

This completes the description of the proposed photon emission process. The main points are: (1) external positional constraints cause, via changes to geometric span, constraints on the internal frequency that the electron can take; (2) an independent constraint is that the electron that is bonded to an atom can take only certain discrete frequencies, these being a consequence of the bonding; (3) the electron is therefore required to dispose of any excess energy, which it does by temporarily dumping it into the external three-dimensional space; (4) doing this creates discrete photon discrete force field structures and the associated supporting structures of reactive ends and fibril. These are extracted from the electron structures. We acknowledge that we have not detailed how this creation might occur at the next deeper level of mechanics. The photon thereafter takes a life of its own and moves in the fabric. Our current explanation for its necessity to move is because it has incomplete discrete force structures and therefore needs to access discrete forces from the fabric to maintain stability, hence moving at the same speed as the fabric. This too points to a still deeper mechanism at work.

\subsection{What has been Achieved?}

This paper makes several novel contributions. The first is that it proposes the identity of internal structures to the photon and electron, and the relationships between those internal variables and the external fields produced by the particules. Particularly important here is the proposed causality whereby bonding constraints affect geometric span of the particule, which affects frequency, and hence constrains the energy that the electron can contain. A second contribution is the proposed differentiation between the photon and electron by the type of their discrete fields. This provides a new way to understand the mechanics and purpose of these fields. Included here is an explanation for the evanescent field, recovery of the exponential and inverse radius squared relationships for the evanescent and EMG fields. Also, an ontologically rich explanation is provided for the fine structure constant $\alpha$. 
The third contribution is the identification of a proposed set of processes for emission and absorption of a photon, at the next lower fundamental level. We have generated a qualitative mechanics for the interaction of the external field with the internal structural variables, so we can start to put answers to the foundational questions of emission and absorption.

\subsection{Implications}

Quantum mechanics originated with the observation that the movement of electrons between orbitals resulted in emission of photons with discrete quanta of energy. This could not be explained by continuum electromagnetic wave-theory. And while quantum mechanics has been effective at quantifying the energy of these emissions, it has not really ever addressed the question of how the transitions occur. It is understandable that $\mathrm{QM}$ would be unable to do this, since it is based on the premise that particles are zero-dimensional points. Nonetheless it is a valid question to ask, and the novel contribution here is showing that a solution does exist in NLHV physics. We also believe that the theory developed here is capable of further extension to explain a number of other photon emission phenomena.

\subsection{Limitations and Implications for Further Research}

This is a conceptual theory, with all the limitations that such theories contain. It does have high fitness in its ability to provide a logically consistent explanation for a wide variety of physical phenomena, including some of the most problematic issues at the foundation of physics. Yet we acknowledge that fitness does not necessarily equate to validity.

At present the theory relies on conjectured differences in the discrete field structures of the photon and electron. While this treatment is sufficient for qualitative purposes, it does not permit quantitative modelling of emission dynamics. This lack of a mathematical formalism is a limitation. It is a consequence of the novel design method applied, rather than an invalidation per se, but a limitation and an opportunity for future work all the same. Intended future work includes the development of a rigorous mathematical formalism. Our preliminary predictions are that a mathematics will need to be developed to symbolise the concept of discrete forces, their orthogonal nature, and their frequency attributes. This is seen as necessary to represent the synchronous interaction (strong force), which dominates all the inter-particle mechanics in this framework. In the photon emission process as envisaged here, the electron dumps excess energy into the fabric via the creation of an evanescent field, and then the subsequent collapse of this field creates the independent photon structures. Consequently we anticipate a need for the formalism to express the interaction between discrete forces and evanescent field. The overall objective of such a mathematical formalism would be to represent the mechanics of photon emission, so that the theory could be quantitative tested against empirical results for photon emission kinematics. The difficulty at this stage is the first part of this process, the creation of a mathematical framework. It is not to be expected that quantum mechanics or the wave-function could be generalised to do this, because the QM mathematics is predicated on a stochastic approximation of what the Cordus theory asserts is a deeper determinism, and hence QM lacks the requisite variability. There may be more to be gained by considering string theories, since they have the requisite higher number of variables and more powerful mathematical constructs.

\section{Conclusions}

We have used the particular non-local hidden-variable solution provided by the Cordus theory to propose a theory for the physical process of photon emission and absorption from the electron. This theory provides some answers to the deep questions of how the electron emerges from, or is absorbed into, the electron. The explanations are in terms of a hypothesised difference in the discrete fields between the photon and electron. The theory provides a new conceptual framework for explaining the process, and hints at yet deeper internal variables and inner-causality. While abandoning the zero-dimensional point paradigm makes this a drastic idea, the resulting Cordus theory provides a physically descriptive explanation for the process of photon-emission and absorption, and has good fitness to explain a wide range of fundamental phenomena.

Quantum mechanics originated with the observation that the movement of electrons between orbitals resulted in emission of photons with discrete quanta of energy. And while quantum mechanics has been effective at quantifying the energy of these emissions, it does not explain how the transitions occur. It is understandable that QM would be unable to do this, since it is based on the premise that particles are zero-dimensional points. Nonetheless it is a valid question to ask. Nor can this be explained by classical continuum electromagnetic wave-theory. The original contribution here is showing that a solution does exist in NLHV physics. Assuming this solution to be valid then the implications would be that particles have internal structure and that quantum mechanics is merely a quantitative statistical summary of a deeper mechanics. 


\section{Notes}

Note 1. It would not work for the electron to instead put the excess energy into the electric field, since this would create an enduring commitment: the electron would feel the constraint when that discrete force interacted with another remote body some time in the future. The electric hyff system is not an energy storage system: it only transmits the force (displacement) when the discrete force reaches a target. By comparison the evanescent field is an energy storage system, and without future liabilities, and therefore a more convenient place for the electron to dump excess energy.

Note 2. If this were true we might expect in some situations that the electron did not change its energisation by a half-cycle increment but rather by whatever fraction is necessary to dispose of the photon energy. However it is more likely that the electron, in many bonding situations, is not free to unilaterally change its phase, because it is synchronised with other particules including the nucleus. Thus if one electron of a bound pair changes phase, the other partner will need to as well. Hence this also provides a mechanism for entanglement.

Note 3. In which case we would expect antielectrons to emit photons in a direction $90^{\circ}$ to that of a similarly constrained electron. This may be testable.

\section{References}

Alexanian, M., \& Bose, S. K. (2006). Two-photon resonance fluorescence. Physical Review A - Atomic, Molecular, and Optical Physics, 74(6). http://dx.doi.org/10.1103/PhysRevA.74.063418

Bell, J. S. (1964). On the Einstein Podolsky Rosen Paradox. Physics, 1(3), 195-200. Retrieved from http://philoscience.unibe.ch/documents/TexteHS10/bell1964epr.pdf

Cohen-Tannoudji, C., Dupont-Roc, J., \& Grynberg, G. (1998). Atom-Photon Interactions: Basic Processes and Applications: Wiley.

de Broglie, L. (1925). Recherches sur la théorie des quanta (Researches on the quantum theory). Annales de Physique, 3(10). Retrieved from http://tel.archives-ouvertes.fr/docs/00/04/70/78/PDF/tel-00006807.pdf

Dirac, P. A. M. (1930). Principles of Quantum Mechanics. Oxford University Press.

FIPS. (1993). Integration Definition for Function Modeling (IDEF0). Retrieved 12 Aug 2003, from http://www.itl. nist.gov/fipspubs/idef02.doc

Groblacher, S., Paterek, T., Kaltenbaek, R., Brukner, C., Zukowski, M., Aspelmeyer, M., \& Zeilinger, A. (2007). An experimental test of non-local realism. Nature, 446(7138), 871-875. http://dx.doi.org/10.1038/ nature 05677

Keller, O. (2000). Space-time description of photon emission from an atom. Physical Review A - Atomic, Molecular, and Optical Physics, 62(2), 022111-022111.

Keller, O. (2006). On the theory of spatial photon localization: Fundamentals and the role of near-field plasma screening.

Leggett, A. (2003). Nonlocal Hidden-Variable Theories and Quantum Mechanics: An Incompatibility Theorem. Foundations of Physics, 33(10), 1469-1493. http://dx.doi.org/10.1023/a:1026096313729

Loudon, R. (2001). The Quantum Theory of Light. Oxford University Press.

Pons, D. J., \& Pons, A. D. (2013). Outer boundary of the expanding cosmos: Discrete fields and implications for the holographic principle The Open Astronomy Journal, 6, 77-89. http://dx.doi.org/10.2174/1874381101306 010077

Pons, D. J., Pons, A. D., \& Pons, A. J. (2013a). Explanation of the Table of Nuclides: Qualitative nuclear mechanics from a NLHV design. Applied Physics Research, 5(6), 145-174. http://dx.doi.org/10.5539/apr.v5 n6p 145

Pons, D. J., Pons, A. D., \& Pons, A. J. (2013b). Synchronous interlocking of discrete forces: Strong force reconceptualised in a NLHV solution Applied Physics Research, 5(5), 107-126. http://dx.doi.org/10.5539/ apr.v5n5107

Pons, D. J., Pons, A. D., \& Pons, A. J. (2013c). Time: An emergent property of matter. Applied Physics Research, 5(6), 23-47. http://dx.doi.org/10.5539/apr.v5n6p23

Pons, D. J., Pons, A. D., \& Pons, A. J. (2014a). Annihilation mechanisms. Applied Physics Research, 6(2), 28-46. http://dx.doi.org/10.5539/apr.v6n2p28 
Pons, D. J., Pons, A. D., \& Pons, A. J. (2014b). Asymmetrical genesis by remanufacture of antielectrons. Journal of Modern Physics, 5, 1980-1994. http://dx.doi.org/10.4236/jmp.2014.517193

Pons, D. J., Pons, A. D., \& Pons, A. J. (2014c). Differentiation of Matter and Antimatter by Hand: Internal and External Structures of the Electron and Antielectron. Physics Essays, 27, 26-35. http://vixra.org/abs/1305. 0157

Pons, D. J., Pons, A. D., \& Pons, A. J. (2015a). Asymmetrical neutrino induced decay of nucleons Applied Physics Research, 7(2), 1-13. http://dx.doi.org/10.5539/apr.v7n2p1 or http://vixra.org/abs/1412.0279

Pons, D. J., Pons, A. D., \& Pons, A. J. (2015b). Hidden variable theory supports variability in decay rates of nuclides Applied Physics Research, 7(3), 18-29. http://dx.doi.org/10.5539/apr.v7n3p18

Pons, D. J., Pons, A. D., \& Pons, A. J. (2015c). Weak interaction and the mechanisms for neutron stability and decay Applied Physics Research, 7(1), 1-11. http://dx.doi.org/10.5539/apr.v7n1p1

Pons, D. J., Pons, A. D., Pons, A. M., \& Pons, A. J. (2012). Wave-particle duality: A conceptual solution from the cordus conjecture. Physics Essays, 25(1), 132-140. http://physicsessays.org/doi/abs/10.4006/0836-1398-25. 1.132

Zavatta, A., Viciani, S., \& Bellini, M. (2005). Single-photon excitation of a coherent state: Catching the elementary step of stimulated light emission. Physical Review A - Atomic, Molecular, and Optical Physics, 72(2). http://dx.doi.org/10.1103/PhysRevA.72.023820

\section{Copyrights}

Copyright for this article is retained by the author(s), with first publication rights granted to the journal.

This is an open-access article distributed under the terms and conditions of the Creative Commons Attribution license (http://creativecommons.org/licenses/by/3.0/). 